\title{
A Survey of the FDA's Adverse Event Reporting System Database Concerning Urogenital Tract Infections and Sodium Glucose Cotransporter-2 Inhibitor Use
}

\author{
Juan Shen · Jincheng Yang $\cdot$ Bin Zhao
}

Received: January 4, 2019 / Published online: April 5, 2019

(C) The Author(s) 2019
Results: On the basis of 37,100 reports, 1628 reports $(4.39 \%$ of total adverse drug reactions, ADRs) were associated with UTIs; among them, the number of UTIs reported for the top four was as follows: canagliflozin, 858 (52.74\%); dapagliflozin, 324 (19.91\%); empagliflozin, 189 (11.62\%); and empagliflozin/metformin, 183 $(11.25 \%)$. Although the number of ADRs reported for these drugs is different, their risk of causing UTIs is determined. Of the reports of SGLT-2i adverse events related to UTIs, $61.73 \%$ occurred in women, which was a much higher proportion than that in men $(28.50 \%)$.

Conclusion: FAERS data were consistent with clinical studies on a strong association between SGLT-2i use and UTIs. The results strongly suggest that female patients are more likely than male patients to experience UTIs when using SGLT-2i.

Keywords: Adverse drug reactions; FAERS; Sodium glucose cotransporter-2 inhibitors; Urogenital tract infections

\section{INTRODUCTION}

Glucose filtered by the kidney is virtually totally reabsorbed in the proximal tubule by sodium glucose cotransporter-2 (SGLT-2) [1]. SGLT-2 inhibitors (SGLT-2i) are attractive, insulin-independent targets for increasing glucose excretion in treatment of type 2 diabetes mellitus 
(T2DM) [2]. SGLT-2i molecules are stable and reliable in reducing blood glucose and weight without increasing the risk of hypoglycemia $[3,4]$. A meta-analysis also showed that SGLT-2i use has significant cardiovascular and renal protective effects that can reduce the incidence of hypoglycemia and acute kidney injury but increase the risk of urogenital infection [5]. This effect is because of the SGLT-2i mechanism in which the glucose concentration in the urogenital region of the patient is increased, resulting in the possibility of bacterial and fungal infections. However, as a result of the short time-to-market for such drugs and the lack of safety evaluation data, large-scale realworld studies are still lacking.

Some countries or organizations have established pharmacovigilance systems, such as the World Health Organization (WHO), European Medicines Agency (EMA), and the US Food and Drug Administration (FDA), for adverse drug reactions (ADRs) monitoring. The FDA's Adverse Event Reporting System (FAERS) is the largest repository of passively reported adverse drug events in the world [6]. It relies on health professionals, consumers, and manufacturers to report all adverse events to the FDA [7]. The purpose of this system is to support the FDA's safety monitoring of postmarketing drugs and therapeutic biologics. This study collected, screened, and statistically analyzed the data in the FAERS, conducted a signal mining analysis of suspicious ADRs generated by SGLT-2i use, and focused on the relevance in urogenital tract infections (UTIs). The common types of UTIs include pyelonephritis, urethritis, cystitis, vulvovaginal fungal infections, vaginal candidiasis, vulvovaginitis, candida balanitis, and penile dermatitis.

Real-world research has emerged after the drug postmarketing, and its influence in the pharmaceutical industry will not be underestimated. It is characterized by selecting treatment based on the patient's actual condition and willingness and conducting long-term evaluation. The sample size is generally larger and the focus is on meaningful outcome treatment. Our statistical results are just based on real-world research. In contrast, randomized controlled trials (RCTs) are usually selected for study under strict inclusion and exclusion criteria. The representativeness and external authenticity of RCTs have certain limitations.

\section{METHODS}

\section{Data Source}

This study used FAERS database data from the first quarter of 2004 to the second quarter of 2018. FAERS is a public voluntary spontaneous reporting database that provides information on adverse events and medication error reports submitted by health professionals, consumers, and manufacturers.

The ASCII data files of the FAERS contain demographic and administrative information (DEMO), drug information (DRUG), adverse events (REAC), patient outcomes (OUTC), report sources (RPSR), therapy start dates and end dates for reported drugs (THER), and indications for use (INDI).

A total of 9,354,226 reports were retrieved from the FAERS. A deduplication procedure was performed according to the FDA's recommendations to select the latest FDA_DT with the same CASEID and select the higher PRIMARYID when CASEID and FDA_DT are both the same, resulting in a reduction in the number of reports to $9,352,387$. On the basis of the PRIMARYID of the deduplicated DEMO, DRUG and REAC were both deduplicated as well.

\section{Drug and Adverse Event Identification}

Since drug names in the FAERS database are registered arbitrarily, MICROMEDEX ${ }^{\circledR}$ (Index Nominum) was utilized as a dictionary for the SGLT-2 drug names (Table 1). Both generic name and brand name were used as keywords for FAERS database retrieval.

In the REAC files, preferred term, PT, means recommended medical terminology describing the event, using the Medical Dictionary for Regulatory Activities (MedDRA). We subtotaled all PTs in the REAC files and found two terms, "UTI" and "urogenital infection", related to UTIs. 
Table 1 Generic names and brand names of antipsychotics in MICROMEDEX ${ }^{\circledR}$

\begin{tabular}{ll}
\hline Generic name & Brand name \\
\hline Canagliflozin & Canaglu $^{\circledR}$, Invokana $^{\circledR}$ \\
Canagliflozin/metformin hydrochloride & Invokamet $^{\circledR}$, Vokanamet $^{\circledR}$ \\
Dapagliflozin propanediol & Edistride $^{\circledR}$, Farxiga $^{\circledR}$, Forxiga $^{\circledR}$, Forziga $^{\circledR}$ \\
Dapagliflozin propanediol/metformin hydrochloride & Ebymect $^{\circledR}$, Xigduo $^{\circledR}$, Xigduo XR $^{\circledR}$ \\
Dapagliflozin propanediol/saxagliptin & Qtern $^{\circledR}$ \\
Empagliflozin & Jardiance $^{\circledR}$ \\
Empagliflozin/linagliptin & Glyxamb $^{\circledR}$ \\
Empagliflozin/metformin hydrochloride & Jardiamet $^{\circledR}$, Jardiance Duo $^{\circledR}$, Synjardy $^{\circledR}$ \\
Ertugliflozin & Steglatro $^{\circledR}$ \\
Ertugliflozin/metformin hydrochloride & Segluromet $^{\circledR}$ \\
Ertugliflozin/sitagliptin & Steglujan $^{\circledR}$ \\
Ipragliflozin & Suglat $^{\circledR}$ \\
Luseogliflozin & Lusef $^{\circledR}$ \\
Tofogliflozin & Apleway $^{\circledR}$, Deberza $^{\circledR}$ \\
\hline
\end{tabular}

\section{Data Mining}

For pharmacovigilance, disproportionality analysis and Bayesian analysis were used to analyze the postmarketing surveillance databases to assess associations between drugs and adverse events. The disproportionality analysis includes the reporting odds ratio (ROR) and the proportional reporting ratio (PRR), whereas the Bayesian analysis includes the Bayesian confidence propagation neural network (BCPNN) and the multi-item gamma Poisson shrinker (MGPS). We used these four algorithms to identify statistical associations between drugs and events in the FAERS. ROR and PRR are more sensitive than Bayesian analyses and have been adopted by Lareb and the European Medicines Agency, respectively. BCPNN and MGPS are more specific than disproportionality and have been adopted by the Uppsala Monitoring Centre and FDA, respectively. The four algorithms' computation and criteria [8-12] are shown as follows: ROR $=$ $a d / c / b, 95 \%$ CI is $e^{\ln (\mathrm{ROR}) \pm 1.96}(1 / a+1 / b+1 /$ $c+1 / d)^{0.5}$ (criteria: 95\% CI $>1$ and $N \geq 2$ );
$\mathrm{PRR}=a(c+d) / c /(a+b), \quad \chi^{2}=\left[(a d-b c)^{2}\right](a+$ $b+c+d) /[(a+b)(c+d)(a+c)(b+d)] \quad$ (criteria: $\mathrm{PRR} \geq 2, \chi^{2} \geq 4$, and $\left.N \geq 3\right)$; with BCPNN algorithms, $\quad \mathrm{IC}=\log _{2} a(a+b+c+d)(a+$ $c)(a+b), 95 \% \mathrm{CI}=e^{\ln (\mathrm{IC}) \pm 1.96(1 / a+1 / b+1 / c+1 / d) 0.5}$ (criteria: IC025 >0); with MGPS algorithms, Empirical Bayesian Geometric Mean (EBGM) = $a(a+b+c+d) /(a+c) /(a+b), \quad 95 \%$ $\mathrm{CI}=e^{\ln (\mathrm{EBGM}) \pm 1.96(1 / a+1 / b+1 / c+1 / d) 0.5} \quad$ (criteria: EBGM05 $>2$ and N $>0$ ). In these algorithms, $a$ is the number of reports with suspect ADRs of the suspect drug; $b$ is the number of reports with the suspect ADRs of all other drugs; $c$ is the number of reports with all other ADRs of the suspect drug; $d$ is the number of reports with all other ADRs of all other drugs; $\mathrm{CI}$ is the confidence interval; $\mathrm{N}$ is the number of co-occurrences of SGLT-2i use and UTIs; $\chi^{2}$ is chi-squared; IC is the information component; ICO25 is a lower limit of the $95 \%$ two-sided CI of the IC. 


\section{Compliance with Ethics Guidelines}

All procedures performed in studies involving human participants were in accordance with the 1964 Helsinki declaration and its later amendments or comparable ethical standards. As this was an observational and retrospective study, and the database is open to the public, this study was exempt from approval by Peking Union Medical College Hospital Institutional Review Board and informed consent was not obtained.

\section{RESULTS}

Overall, we identified a total of 1628 cases of SGLT-2i use with UTIs in the FAERS database. A total of 80,548 cases related to UTIs and a total of 37,100 adverse events related to SGLT-2i use were documented in the FAERS between the first quarter of 2004 and the second quarter of 2018. Among them, $4.39 \%(1628 / 37,100)$ had SGLT-2i adverse events related to UTIs; $61.73 \%$ of these events occurred in women, $28.50 \%$ occurred in men, and gender was unknown in $9.77 \%$ of the events. All of the reports involved adult patients (aged $>18$ years), and the average age was 60.17 years (Table 2 ).

No co-occurrences of SGLT-2i use and UTIs were reported for dapagliflozin/saxagliptin (approved in the USA, February 2017), ertugliflozin/metformin (approved in the USA, December 2017), ertugliflozin/sitagliptin (approved in the USA, December 2017), ipragliflozin (approved in Japan, April 2014), luseogliflozin (approved in Japan, May 2014), or tofogliflozin (approved in Japan, March 2014), and only three co-occurrences of SGLT-2i use and UTIs were reported for ertugliflozin (approved in the USA, December 2017).

The statistical data on SGLT-2i-associated UTIs are listed in Table 3. An association with UTIs was suggested for every SGLT-2i except the ones mentioned above.

\section{DISCUSSION}

The WHO definition of pharmacovigilance is the scientific activity of discovering, evaluating, understanding, and preventing adverse drug effects or other drug-related problems. Pharmacovigilance runs through the entire life cycle of drug development. In the postmarketing surveillance phase, the important challenge of pharmacovigilance is how to collect and analyze observational data for the drugs and obtain a more persuasive power. The conclusion is that the ADR signals are generated and tested. Therefore, choosing a rapid and effective signal detection method can provide valuable signals for drug risk management and clinical evaluation to facilitate the timely and accurate detection of dangerous drugs that seriously endanger human health and minimize its harm to humans.

This study draws the conclusion that SGLT$2 \mathrm{i}$ use does have adverse reactions with regards to UTIs. In December 2015, the FDA reported 19 cases of life-threatening blood infections and kidney infections that started as UTIs with the SGLT-2i reported to the FAERS from March 2013 through October 2014 [13]. All 19 patients were hospitalized, and a few required admission to the intensive care unit or dialysis to treat kidney failure. Clinical studies have confirmed that compared with placebo or other antidiabetic drug administration, SGLT-2i use caused UTIs that were mild or moderate in intensity $[14,15]$. However, only one study showed that SGLT-2i use did not increase the risk of UTIs, except for high-dose (10 mg/day) dapagliflozin in patients with T2DM [16].

However, monitoring adverse events can help us discover rare but potentially serious adverse reactions and provide an important basis for follow-up prevention. This effect may only be discovered after SGLT-2i are widely used in the clinic. It is very difficult to achieve this goal only through experimental research with limited sample sizes. Therefore, this study provides a reference for confirming the occurrence of UTIs associated with SGLT-2i use through postmarketing surveillance studies of this class of drugs.

UTIs can be stratified as healthcare-associated urogenital tract infection (HAUTI) and community-associated urogenital tract infection (CAUTI). The incidence of UTIs in developing countries is higher than that in the USA 
Table 2 Gender and age for SGLT-2i use

\begin{tabular}{|c|c|c|c|c|c|c|c|}
\hline & \multicolumn{3}{|l|}{ Gender } & \multicolumn{4}{|l|}{ Age } \\
\hline & Male $(n, \%)$ & Female $(n, \%)$ & $\begin{array}{l}\text { Unknown (n, } \\
\%)\end{array}$ & $\begin{array}{l}<18 \\
(n)\end{array}$ & $\begin{array}{l}18-65 \\
(n)\end{array}$ & $\begin{array}{l}>65 \\
(n)\end{array}$ & $\begin{array}{l}\text { Average } \\
\text { (years) }\end{array}$ \\
\hline Canagliflozin & $\begin{array}{l}222 \\
\quad(25.87 \%)\end{array}$ & $538(62.70 \%)$ & $98(11.42 \%)$ & 0 & 292 & 131 & 59 \\
\hline $\begin{array}{l}\text { Canagliflozin/ } \\
\text { metformin }\end{array}$ & $2(8.70 \%)$ & $18(78.26 \%)$ & $3(13.04 \%)$ & 0 & 11 & 2 & 56 \\
\hline Dapagliflozin & $95(29.23 \%)$ & $198(60.92 \%)$ & $32(9.85 \%)$ & 0 & 125 & 62 & 60 \\
\hline $\begin{array}{l}\text { Dapagliflozin/ } \\
\text { metformin }\end{array}$ & $9(37.50 \%)$ & $15(62.50 \%)$ & $0(0.00 \%)$ & 0 & 7 & 7 & 62 \\
\hline $\begin{array}{c}\text { Dapagliflozin/ } \\
\text { saxagliptin }\end{array}$ & $0(-)$ & $0(-)$ & $0(-)$ & 0 & 0 & 0 & - \\
\hline Empagliflozin & $68(35.98 \%)$ & $109(57.67 \%)$ & $12(6.35 \%)$ & 0 & 71 & 56 & 63 \\
\hline $\begin{array}{c}\text { Empagliflozin/ } \\
\text { linagliptin }\end{array}$ & $4(17.39 \%)$ & $17(73.91 \%)$ & $2(8.70 \%)$ & 0 & 8 & 4 & 64 \\
\hline $\begin{array}{l}\text { Empagliflozin/ } \\
\text { metformin }\end{array}$ & $62(33.88 \%)$ & $109(59.56 \%)$ & $12(6.56 \%)$ & 0 & 69 & 51 & 62 \\
\hline Ertugliflozin & $2(66.67 \%)$ & $1(33.33 \%)$ & $0(0.00 \%)$ & 0 & 1 & 0 & 46 \\
\hline Ertugliflozin/metformin & $0(-)$ & $0(-)$ & $0(-)$ & 0 & 0 & 0 & - \\
\hline Ertugliflozin/sitagliptin & $0(-)$ & $0(-)$ & $0(-)$ & 0 & 0 & 0 & - \\
\hline Ipragliflozin & $0(-)$ & $0(-)$ & $0(-)$ & 0 & 0 & 0 & - \\
\hline Luseogliflozin & $0(-)$ & $0(-)$ & $0(-)$ & 0 & 0 & 0 & - \\
\hline Tofogliflozin & $0(-)$ & $0(-)$ & $0(-)$ & 0 & 0 & 0 & - \\
\hline
\end{tabular}

and Europe [17]. This may be related to the different levels of healthcare development. It is also reported in the literature that the incidence of UTIs in Japan is lower than that in South Korea, Taiwan, and substantially lower than those of developing countries. The national surveillance programs may lead to a sustained decrease of incidence in Japan [18]; but, we also cannott deny that Japan is a country that has good washroom culture and toilet habits. Previous studies showed that the incidence of UTIs will be greatly increased in patients with T2DM [19]. In our study, we further found that 1628 T2DM cases had SGLT-2i related UTIs. The vast majority of reports were from the USA (1268), which may be related to the database being from the FDA. Some reports are from the UK (44), Canada (41), Germany (39), Japan (39), and China (41). Only a few reports were from developing countries, such as Vietnam (2) and Thailand (2). We cannot judge the UTI incidence by the number of reports. The reason for this phenomenon is SGLT-2i: their use in economically underdeveloped areas is still limited, and the attention to ADRs in these areas needs to be improved.

A meta-analysis of RCTs in 2016 showed that canagliflozin, dapagliflozin, and empagliflozin were associated with a significantly higher risk of genital infections compared with placebo and other active treatments [20]; however, this conclusion is derived from the results of RCTs. 
Table 3 Signal detection for SGLT-2i-associated UTIs

\begin{tabular}{|c|c|c|c|c|c|}
\hline & $N$ & $\begin{array}{l}\text { ROR }(95 \% \\
\text { two-sided CI) }\end{array}$ & $\operatorname{PRR}\left(\chi^{2}\right)$ & $\begin{array}{l}\text { IC }(95 \% \\
\text { two-sided CI })\end{array}$ & $\begin{array}{l}\text { EBGM }(95 \% \\
\text { one-sided CI })\end{array}$ \\
\hline Canagliflozin & 858 & $5.88(5.49,6.30)$ & $5.83(3275.52)$ & $63.32(59.10,67.84)$ & $5.60(5.23)$ \\
\hline Canagliflozin/metformin & 23 & $3.66(2.41,5.54)$ & $3.66(43.03)$ & $53.52(35.34,81.07)$ & $3.57(2.36)$ \\
\hline Dapagliflozin & 324 & $5.80(5.19,6.49)$ & $5.78(1221.07)$ & $60.52(54.12,67.68)$ & $5.55(4.97)$ \\
\hline Dapagliflozin/metformin & 24 & $5.93(3.93,8.94)$ & $5.93(93.52)$ & $52.98(35.15,79.85)$ & $5.69(3.77)$ \\
\hline Dapagliflozin/saxagliptin & 0 & - & - & - & - \\
\hline Empagliflozin & 189 & $4.27(3.70,4.94)$ & $4.27(455.92)$ & $59.39(51.35,68.68)$ & $4.15(3.59)$ \\
\hline Empagliflozin/linagliptin & 23 & $4.18(2.76,6.33)$ & $4.18(53.61)$ & $53.34(35.18,80.86)$ & $4.062 .68)$ \\
\hline Empagliflozin/metformin & 183 & $4.11(3.55,4.77)$ & $4.11(415.43)$ & $59.35(51.20,68.79)$ & $4.00(3.45)$ \\
\hline Ertugliflozin & 3 & $14.39(4.33,47.79)$ & $14.39(33.22)$ & $45.79(13.79,152.08)$ & $12.90(3.88)$ \\
\hline Ertugliflozin/metformin & 0 & - & - & - & - \\
\hline Ertugliflozin/sitagliptin & 0 & - & - & - & - \\
\hline Ipragliflozin & 0 & - & - & - & - \\
\hline Luseogliflozin & 0 & - & - & - & - \\
\hline Tofogliflozin & 0 & - & - & - & - \\
\hline
\end{tabular}

$N$ number of co-occurrences of SGLT-2i use and UTIs, ROR reporting odds ratio, $C I$ confidence interval, $P R R$ proportional reporting ratio, $\chi^{2}$ chi-squared, $I C$ information component, $E B G M$ empirical Bayesian geometric mean

In our real-world study, we also found that canagliflozin, dapagliflozin, empagliflozin, and their combination have a risk of UTIs, which are consistent. Similarly, from the available data, we are still unable to judge whether the above drugs have differences in the incidence of UTIs. Therefore, we recommend that the medical staff should pay attention to the UTIs problem when using SGLT-2i.

Although female patients, particularly those aged 16-64 years, are significantly more likely to experience UTIs than male patients [21], urinary infections frequently occur in both genders and across all age groups [22], and specific situations such as pregnancy, use in the elderly, menstruation, catheterization, diabetes, and other opportunities to induce infection are especially relevant [23]. The results of our study confirmed that the incidence of SGLT-2i-associated UTIs in women was significantly higher than that in men. After analyzing the reasons, we believe that this effect may be related to the following factors: (1) women have special physiology and anatomy since the urogenital tract environment and $\mathrm{pH}$ value cause UTIs to occur more easily; (2) female urogenital tract blood circulation is different from that of men, i.e., when using the same doses, there may be a difference in the local blood drug concentration; and (3) the symptoms are mild or asymptomatic after early genitourinary infection in women and are often ignored and cannot be treated promptly. Considering all of the above factors, our results confirm that stratification of isolates from unselected patients on the basis of gender can improve reasonable SGLT-2i use and reduce the incidence of ADRs. However, patient age is not a significant factor in determining SGLT-2i-associated UTIs in our research.

Overall, in order to avoid the occurrence of UTIs, we suggested to ask for a medical history before use. These infections were more frequent in the 6 months post initiation [24]. In the course of use, if the patient has symptoms of 
UTIs, seek medical attention. For patients using SGLT-2i, it is recommended to pay attention to the hygiene of the individual genital area, drink plenty of water, keep the urine flowing, and reduce the incidence of infection.

Although the data mining techniques used in this study have many advantages in the study of adverse drug reaction signals, we also need to note that this technology cannot solve all problems in the detection and analysis of adverse drug reaction signals. The signals from spontaneous reporting systems were only used in qualitative research. Therefore, unavoidably, this study has certain limitations. First, data mining technology fails to fully reflect all clinical information from patients. Detailed information is also needed from clinical follow-up and other surveys to verify data mining hypotheses. Second, data mining technology cannot remedy the inherent limitations of ADR reporting systems, such as underreporting, false reporting, incomplete reporting, inaccuracy, and arbitrariness. Third, it is difficult to quantify the adverse reaction signals of UTIs from the number of ADRs. Finally, the influence of glycemic control factors such as HbA1c cannot be excluded.

\section{CONCLUSION}

The primary aim of our study is compared the frequency of UTIs for each SGLT-2i. Analysis of FAERS ADR signal data mining confirmed a statistically significant association between SGLT-2i use and UTIs. There is no difference among canagliflozin, canagliflozin/metformin, dapagliflozin, dapagliflozin/metformin, empagliflozin, empagliflozin/linagliptin, and empagliflozin/metformin in causing UTIs. In addition, the data strongly suggest that female patients are more likely than male patients to experience UTIs when using an SGLT-2i. Large, well-organized epidemiologic studies are needed to confirm these findings.

\section{ACKNOWLEDGEMENTS}

We thank all participants of the study.
Funding. This study was supported in part by the National Natural Science Foundation of China (No. 81603218). The article processing charges were funded by the authors.

Authorship. All named authors meet the International Committee of Medical Journal Editors (ICMJE) criteria for authorship for this article, take responsibility for the integrity of the work as a whole, and have given their approval for this version to be published.

Authorship Contributions. Bin Zhao performed the design of this study. Juan Shen and Jincheng Yang collected data and wrote/edited the manuscript.

Disclosures. The authors Juan Shen, Jincheng Yang, and Bin Zhao have nothing to disclose.

Compliance with Ethics Guidelines. All procedures performed in studies involving human participants were in accordance with the 1964 Helsinki declaration and its later amendments or comparable ethical standards. As this was an observational and retrospective study, and the database is open to the public, this study was exempt from approval by Peking Union Medical College Hospital Institutional Review Board and informed consent was not obtained.

Open Access. This article is distributed under the terms of the Creative Commons Attribution-NonCommercial 4.0 International License (http://creativecommons.org/licenses/ by-nc/4.0/), which permits any noncommercial use, distribution, and reproduction in any medium, provided you give appropriate credit to the original author(s) and the source, provide a link to the Creative Commons license, and indicate if changes were made.

\section{REFERENCES}

1. Wright EM, Loo DD, Hirayama BA. Biology of human sodium glucose transporters. Physiol Rev. 2011;91(2):733-94. 
2. Gallo LA, Wright EM, Vallon V. Probing SGLT2 as a therapeutic target for diabetes: basic physiology and consequences. Diab Vasc Dis Res. 2015;12(2):78-89.

3. Ceylan AF, Ren SY. Sodium glucose co-transporter 2 inhibitors: beyond glucose lowering. Curr Drug Targets. 2018;19(9):1045-50.

4. Zhang L, et al. Efficacy and safety of sodium-glucose cotransporter 2 inhibitors in patients with type 2 diabetes and moderate renal function impairment: A systematic review and meta-analysis. Diabetes Res Clin Pract. 2018;140:295-303.

5. Radholm $\mathrm{K}$, et al. Effects of sodium-glucose cotransporter-2 inhibitors on cardiovascular disease, death and safety outcomes in type 2 diabetes-a systematic review. Diabetes Res Clin Pract. 2018;140:118-28.

6. Rodriguez EM, Staffa JA, Graham DJ. The role of databases in drug postmarketing surveillance. Pharmacoepidemiol Drug Saf. 2001;10(5):407-10.

7. Weiss-Smith S, et al. The FDA drug safety surveillance program: adverse event reporting trends. Arch Intern Med. 2011;171(6):591-3.

8. van Puijenbroek EP, et al. A comparison of measures of disproportionality for signal detection in spontaneous reporting systems for adverse drug reactions. Pharmacoepidemiol Drug Saf. 2002;11(1):3-10.

9. Evans SJ, Waller PC, Davis S. Use of proportional reporting ratios (PRRs) for signal generation from spontaneous adverse drug reaction reports. Pharmacoepidemiol Drug Saf. 2001;10(6):483-6.

10. Bate A, et al. A Bayesian neural network method for adverse drug reaction signal generation. Eur J Clin Pharmacol. 1998;54(4):315-21.

11. Szarfman A, Machado SG, O'Neill RT. Use of screening algorithms and computer systems to efficiently signal higher-than-expected combinations of drugs and events in the US FDA's spontaneous reports database. Drug Saf. 2002;25(6):381-92.

12. Sakaeda T, et al. Data mining of the public version of the FDA Adverse Event Reporting System. Int J Med Sci. 2013;10(7):796-803.

13. FDA Drug Safety Communication: FDA revises labels of SGLT2 inhibitors for diabetes to include warnings about too much acid in the blood and serious urinary tract infections. 2015. https://www. fda.gov/Drugs/DrugSafety/ucm475463.htm. Accessed 17 May 2018

14. Thewjitcharoen $\mathrm{Y}$, et al. Effectiveness of long-term treatment with SGLT2 inhibitors: real-world evidence from a specialized diabetes center. Diabetol Metab Syndr. 2017;9:96.

15. Goda M, et al. Safety and efficacy of canagliflozin in elderly patients with type 2 diabetes mellitus: a 1-year post-marketing surveillance in Japan. Curr Med Res Opin. 2018;34(2):319-27.

16. Puckrin R, et al. SGLT-2 inhibitors and the risk of infections: a systematic review and meta-analysis of randomized controlled trials. Acta Diabetol. 2018;55(5):503-14.

17. Tandogdu Z, Wagenlehner FM. Global epidemiology of urinary tract infections. Curr Opin Infect Dis. 2016;29(1):73.

18. Chiang $\mathrm{CH}$, et al. Healthcare-associated infections in intensive care units in Taiwan, South Korea, and Japan: recent trends based on national surveillance reports. Antimicrob Resist Infect Control. 2018;7:129.

19. Nichols GA, et al. Prevalence and incidence of urinary tract and genital infections among patients with and without type 2 diabetes. J Diabetes Complications. 2017;31(11):S1056872717306633.

20. Li D, et al. Urinary tract and genital infections in patients with type 2 diabetes treated with sodiumglucose co-transporter 2 inhibitors: a meta-analysis of randomized controlled trials. Diabetes Obes Metab. 2017;19(3):348.

21. Harrington RD, Hooton TM. Urinary tract infection risk factors and gender. $\mathrm{J}$ Gend Specif Med. 2000;3(8):27-34.

22. Litwin MS, et al. Urologic diseases in America project: analytical methods and principal findings. J Urol. 2005;173(3):933-7.

23. Choe HS, et al. Summary of the UAA-AAUS guidelines for urinary tract infections. Int $\mathrm{J}$ Urol. 2018;25(3):175-85.

24. Gadzhanova S, Pratt N, Roughead E. Use of SGLT2 inhibitors for diabetes and risk of infection: analysis using general practice records from the NPS MedicineWise MedicineInsight program. Diabetes Res Clin Pract. 2017;130:180-5. 